Research Paper:

\title{
Effect of Tai Chi Exercise on the Stress of Elderly Women crosshark With Hypertension
}

\author{
Elnaz Talebi $^{1}$, Farideh Bastani ${ }^{1 *} \bigcirc$, Hamid Haqhani ${ }^{2}$
}

1. Department of Geriatric Nursing, School of Nursing \& Midwifery, Iran University of Medical Sciences, Tehran, Iran. 2. Department of Biostatistics, School of Health, Iran University of Medical Sciences, Tehran, Iran.

\begin{tabular}{|l|l|}
\hline $\begin{array}{c}\text { use your device to scan } \\
\text { and read the article online }\end{array}$ & $\begin{array}{l}\text { Cltation: Talebi, E., Bastani, F., \& Haqhani, H., 2017. Effect of Tai Chi Exercise on the Stress of Elderly Women With } \\
\text { Hypertension. Journal of Client-Centered Nursing Care, 3(4), pp. 263-268. https://doi.org/10.32598/jccnc.3.4.263 }\end{array}$ \\
\hline
\end{tabular}

Article info:

Received: 14 Apr 2017

Accepted: 23 Aug 2017
Keywords:

Elderly, Stress, Hypertension, Tai Chi

\begin{abstract}
A B S T RA C T
Background: Hypertension or high blood pressure is one of the most common chronic diseases among the geriatrics having a strong association with psychological factors such as perceived stress. Aerobic exercises are effective in the prevention and treatment of hypertension and stress management. In the same regard, this paper is an attempt to investigate the effect of Tai Chi exercise on the stress of Iranian older women with hypertension.

Methods: This clinical trial with pre and posttest quasi-experimental design was conducted on 64 elderly women with hypertension at two elderly care centers in Tehran. This study included 8 -form Tai Chi exercise for a period of six weeks. For collecting data, two questionnaires with demographic characteristics and the Perceived Stress Scale (PSS) were used, and for analyzing data, descriptive and inferential statistics were employed in SPSS-PC V21 software.

Results: The results showed that there was no significant difference in perceived stress in both experimental and control groups, before and after the intervention. The results of repeated measures ANOVA also showed that the mean of perceived stress score before and after intervention was not statistically significant $(\mathrm{P}=0.557$ and 0.489$)$.

Conclusion: Since the 8-form Tai Chi exercise is a safe intervention for the elderlies has led to a significant difference in the level of perceived stress among the elderly women, further studies are suggested to find a suitable and effective style of this exercise.
\end{abstract}

\section{Background}

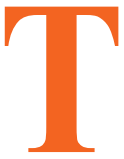

he increase in life expectancy, as well as growing older population in the world, is a major challenge. According to a report by the World Health Organization (2013), between 2000 and 2050, the proportion of the world's population over 60 years will double from about $11 \%$ to $22 \%$, and it is expected to increase from 605 million to 2 billion over the same period. Aged people are confronted with several disabilities and limitations. Among these, hypertension or high blood pressure is considered as an important disease (Lloyd Sherlock et al. 2014). Stress is one of the major factors in hyperten-

\footnotetext{
* Corresponding Author:
}

Farideh Bastani, PhD

Address: Department of Geriatric Nursing, School of Nursing \& Midwifery, Iran University of Medical Sciences, Tehran, Iran.

Tel: +98 (936) 5220789

E-mail: faridehbastani@yahoo.com; bastani,f@iums.ac.ir 
sion (Hapunda et al. 2015) and the mean stress intensity in people with hypertension is significantly higher than those who are not affected with the disease (Vahedian Azimi et al. 2012). It is also important to note that the perceived stress level in women is much higher than that in men. Regular aerobic exercises are one of the basic strategies for the prevention and treatment of hypertension (Lan et al. 2013).

One of the best exercises in this field is called Tai Chi, a multifactorial sport and an elderly-friendly solution according to American Society of Aging (Pho et al. 2012). Tai Chi is a traditional Chinese mind-body exercise. In Tai Chi, smooth and rhythmic movements are linked, causing the transfer of body weight from one leg to other resulting in better balance (Lan et al. 2013). Tai Chi exercise has several forms that vary in different situations (Azimzadeh, Hosseini \& Nourozi Tabrizi 2013). A simplified 8-form program of Tai Chi which is derived from a complex 24-form, allows the elderlies to perform it either by standing or sitting, and let them enjoy Tai Chi exercise without having to worry about remembering different movements, an important method of controlling their stress (Lee 2017).One of the researches on Tai Chi exercise conducted by Taylor-Piliae (2014) showed that Tai Chi Quan is a safe form of exercise to prevent and manage cardiovascular disease with no side effects. They suggested that the extent and routine of Tai Chi (number of sessions, frequency, and duration) are factors that can influence the outcomes of this exercise. Yeh et al. (2008), in a systematic review, investigated the effect of Tai Chi exercise on blood pressure. They concluded that Tai Chi may reduce blood pressure and serve as a practical, non-pharmacologic method for hypertension management. According to them, there are different forms of Tai Chi. Biglari et al. (2016) studied the Effect of 8-Week Tai Chi Quan exercise and walking on cardiovascular-related indicators among Iranian elderly women. Their results indicated that Tai Chi Quan training can be recommended to people who have not enough mobility to exercise, low cost, simplicity, and its beneficial therapeutic properties.

Considering previous studies on Tai Chi exercise and lack of research on its effect on the stress of older women as a vulnerable group, this paper is aimed to study the effect of Tai Chi exercise on the perceived stress of older women suffering from hypertension.

\section{Materials and Methods}

This study is a clinical trial with pre and posttest quasiexperimental design. Study population consists of geriatric women with hypertension in elderly care centers in Tehran under the supervision of welfare organization.
Multi-stage sampling method was applied. Firstly, the names of all elderly care centers which were introduced by the Welfare Organization and their readiness to carry out the study, were placed inside the envelope; then the names of the two centers were randomly taken out (Arad and Tohid elderly centers). Next, from these two centers, by tossing a coin, the Arad center was selected for the test group and Tohid center for the control group. Inclusion criteria for the older women who, according to the definition of the World Health Organization, should be over 60 years having no cognitive impairment by using Abbreviated Mental Test (AMT) instrument, having high blood pressure with no history of depression or anxiety disorder according to the patient or her medical record, no drug addiction or mediation or Tai Chi exercises.

At both the centers, Tohid and Arad, informed consent was taken from the study subjects (which came under the inclusion criteria) and were explained about the goals and stages of the research. The elderlies were examined with the help of cognitive impairment test and after ensuring that they had none, a demographic data form surveying age, weight, height, body mass index, duration of hypertension, underlying disease, marital status, educational level, economic situation and use of mobility aids was completed by the participants. To measure the stress level, Perceived Stress Scale (PSS) was used that was developed by Cohen et al. (1983). This tool is very suitable for measuring the people's perception of stress against the unpredictable and uncontrollable events of life. It has 14 items based on 4-point Likert scale rated as $\mathrm{O}=$ never, 1 = almost never, 2 = sometimes, 3 = fairly often, and $4=$ very often. Scores are obtained by reversing the responses to the positively stated items (items 4, 5, 6, $7,9,10,13)$. In total, scores are from zero to 56 . Reliability and validity of this tool have been reported by using Cronbach's alpha coefficient ranging from 0.74 to 0.93 .

The sample size was determined as $\mathrm{n}=38$ at $95 \%$ confidence level and $80 \%$ test strength in each, test and control group. Due to the long duration of intervention and the lack of familiarity of the elderlies with this exercise, three subjects in the test group due to lack of interest in continuing the Tai Chi exercise, two subjects due to cold and weakness, and one subject because of a stroke were excluded from intervention. In the control group, five elderly women said that they were not interested in filling out a post-test questionnaire, and one woman died. Finally, data from 32 samples in each, test and control group, were analyzed.

Intervention included 8-form Tai Chi exercise for thrice a week sessions, each for 40 mins, for six weeks. It should be noted that the elderly carried out Tai Chi ex- 
ercise under the supervision of a researcher with Tai Chi certification. Also, the exercise was performed in the hall which can accommodate all the elderly women together. The details of the intervention are as following:

Five minutes for warm-up which included relaxation and balancing exercises (by considering the ability of each elderly woman);

20-30 minutes for performing 1-8 movements of Tai Chi as following hand and body positions by listening to Tai Chi music: $1=$ Commencing form where both hands rise to shoulder level; $2=$ Curving back arms; $3=$ Stepping sideways and moving arms; $4=$ Moving hands like clouds; $5=$ Diagonal strides; $6=$ Standing on one leg; $7=$ Stepping and pushing; and $8=$ Closing form where both hands fall to the side and left leg drawn to the right leg.

Five minutes for cooling down involving deep breathing and muscular relaxation.

The control group did not receive any special intervention apart from the routine care. Immediately after com- pleting six weeks of intervention, the perceived stress questionnaire was completed by both test and control groups. Data were analyzed using SPSS V. 21 software. Descriptive statistics (frequency, percentage, mean and standard deviation) were used to analyze the data and to determine the statistical significance inferential statistics (Chi-square, Fisher's exact test, independent t-test, paired t-test, and repeated measures ANOVA) were used.

\section{Results}

The results of the demographic analysis showed that the participants of both the groups had no significant difference in terms of age, weight, height, body mass index, duration of hypertension, underlying disease, marital status, educational level, economic situation and use of mobility aids (Table 1).

The comparison of results for the perceived stress was done for the participants in both the groups, test and control. The pre-intervention stage, by independent t-test, showed that the two groups did not have any significant statistical difference $(\mathrm{P}=0.575)$. The mean score of stress, before intervention, in the test

Table 1. Demographic characteristics of study participants

\begin{tabular}{|c|c|c|c|c|c|c|}
\hline \multirow{2}{*}{ Variables } & \multirow{2}{*}{ Group } & \multicolumn{2}{|c|}{ Test Group } & \multicolumn{2}{|c|}{ Control Group } & \multirow{2}{*}{$\mathbf{P}$} \\
\hline & & $\mathbf{N}$ & $\%$ & $\mathbf{N}$ & $\%$ & \\
\hline \multirow{4}{*}{ Age } & $60-64$ years & 6 & 18.8 & 5 & 15.8 & \multirow{4}{*}{0.555} \\
\hline & $65-69$ years & 14 & 43.8 & 14 & 43.8 & \\
\hline & 70-74 years & 4 & 12.5 & 7 & 21.9 & \\
\hline & $>75$ years & 8 & 25 & 6 & 18.8 & \\
\hline \multirow{4}{*}{ Educational level } & Unlettered & 7 & 21.9 & 5 & 15.6 & \multirow{4}{*}{0.676} \\
\hline & Lettered & 21 & 65.6 & 21 & 65.6 & \\
\hline & Secondary school & 4 & 12.5 & 4 & 12.5 & \\
\hline & Academic & 0 & 0 & 2 & 6.2 & \\
\hline \multirow{4}{*}{ Duration of hypertension } & $<25$ months & 14 & 43.8 & 8 & 25 & \multirow{4}{*}{0.353} \\
\hline & $25-74$ months & 8 & 25 & 6 & 18.8 & \\
\hline & $75-125$ months & 6 & 18.8 & 16 & 50 & \\
\hline & $>125$ months & 4 & 12.5 & 5 & 15.6 & \\
\hline \multirow{3}{*}{ Marital status } & Married & 17 & 53.1 & 21 & 65.6 & \multirow{3}{*}{0.446} \\
\hline & Divorced & 1 & 3.1 & 0 & 0 & \\
\hline & Widowed & 14 & 43.8 & 11 & 34.4 & \\
\hline \multirow{3}{*}{ Economic situation } & Good & 1 & 3.1 & 15 & 46.9 & \multirow{3}{*}{0.001} \\
\hline & Fair & 22 & 68.8 & 16 & 50 & \\
\hline & Poor & 9 & 28.1 & 1 & 3.1 & \\
\hline
\end{tabular}


Table 2. Mean \pm SD and ANOVA results of the perceived stress before and after the intervention in both the study groups

\begin{tabular}{ccccc}
\hline & Test Group & Control Group & Repeated Measures ANOVA \\
\cline { 2 - 4 } & Mean \pm SD & Mean \pm SD & Intra-group & Inter-group \\
\hline Before intervention & $25.16 \pm 7.56$ & $26.45 \pm 10.49$ & $\mathrm{~F}=10.27$ & $\begin{array}{c}\text { Greenhouse--Geisser } \\
\text { test } \\
\mathrm{F}=2.54\end{array}$ \\
After intervention & $23.84 \pm 6.64$ & & $\mathrm{P}=0.489$ & 0.115 \\
Variations & $-1.31 \pm 6.49$ & $25.44 \pm 9.87$ & $\begin{array}{c}\mathrm{t}=0.208 \\
\mathrm{df}=62 \\
\mathrm{P}=0.836\end{array}$ \\
\hline
\end{tabular}

Table 3. Mean \pm SD and paired t-test results of the perceived stress before and after intervention in both study groups

\begin{tabular}{ccc}
\hline & Test Group & Control Group \\
\cline { 2 - 3 } & Mean \pm SD & Mean \pm SD \\
\hline Before intervention & $25.16 \pm 7.56$ & $26.45 \pm 10.49$ \\
After intervention & $23.84 \pm 6.64$ & $25.44 \pm 9.87$ \\
Paired t-test & $t=1.144, d f=31$ & $t=0.130$ \\
& $P=0.262$ & $d f=31$ \\
$P=0.267$
\end{tabular}

Client-Centered Nursing Care

group was 25.16 and in the control group was 26.45 . Independent t-test results also showed that after intervention, the mean score of stress in the test group was 23.84 and in the control group was 25.44 , but there was no significant difference between the two groups after the intervention. Also, the results of ANOVA with repeated measures (inter-group effect) showed that the mean score of perceived stress before and after the intervention was statistically insignificant $(\mathrm{P}=0.557)$. For intra-group effect, the mean score of the two groups had an insignificant statistical difference $(\mathrm{P}=$ 0.489) (Table 2). Independent T-test also showed that there was no significant difference in perceived stress variations in both groups $(\mathrm{P}=0.836)$. Overall, there was no significant difference in the perceived stress in test group ( $\mathrm{P}=0.262)$ and control group $(\mathrm{P}=0.267)$ before and after the intervention (Table 3 ).

\section{Discussion}

The aim of this study was to investigate the effects of Tai Chi exercise on the perceived stress of elderly women with hypertension. The mean age of elderly women with hypertension in the two groups, test (71 years) and control (70 years), was not statistically significant $(\mathrm{P}=$ $0.555)$. Also, the highest age frequency of elderlies was in the range of 65-69 years. It should be noted that these mean ages are below the younger aged group ranging from 60 to 74 years old. Regarding the Body Mass Index (BMI), BMI was 23 for test group, and it was 22 for control group, presenting that there was no significant difference between the two groups $(\mathrm{P}=0.579)$. According to the division of the body mass index the elderly in this study are in the normal range, but it should be noted that the mean BMI of women is significantly higher than that of men. On the other hand, BMI is related to blood pressure therefore in hypertensive patients it is significantly more than those with normal blood pressure.

There was no significant difference between the two groups regarding the duration of hypertension $(\mathrm{P}=$ $0.353)$. In the test group, the hypertension duration for $43.8 \%$ of women was 25 months and less, while in the control group, for $50 \%$ of them it was between $75-125$ months. The two groups did not have any significant difference with respect to the underlying disease, history of the disease and were homogeneous ( $\mathrm{P}=0.106) .59 .4 \%$ in the test group and $78.1 \%$ in the control group had underlying diseases other than hypertension. Participants reported the abnormalities in the cardiovascular system, liver and endocrine system, musculoskeletal system as well as neurological, immune and visual systems which 
indicated the diverse diseases that the elderlies were involved with. This would result in the exponential growth of health costs (Rasel \& Ardalan 2007).

There were insignificant differences between the two groups in terms of educational level $(\mathrm{P}=0.676)$. Sixtysix percent of patients in each group were literate enough to read and write. The mean of stress severity in people with different educational levels had a significant difference with each other. There was a significant difference between the two groups in terms of economic status $(\mathrm{P}$ $<0.001)$ and more than half of the participants had a fair economic status. It seems that since the participants in this study were from the two private elderly care centers in Tehran where it requires a fair income level in order to pay the tuition; this statistical finding can be acceptable. Overall, it was concluded that there were no significant differences in the perceived stress in both test and control groups, before and after the intervention.

Several studies have been conducted over the Tai Chi exercises, some of these studies have shown results that substantiate our findings, and with others, there were no agreements. Lee et al. (2017) investigated the effects of Tai Chi on waist circumference and blood pressure of 68 elderly women aged 65 years. Simplified Yang style 24form Tai Chi was used as the intervention, which was conducted for 5 sessions per week (60 minutes per session), for 6 weeks. They concluded that Tai Chi can be used as an effective intervention to improve waist circumference and blood pressure in the elderly. In another study by Kim et al. (2016), the mental-attention Tai Chi effect on 64 Chinese and non-Chinese adults (51-87 years old) was investigated. Their results suggested that Tai Chi can improve mental-attention, vigilance and executive control, when participants are sufficiently motivated to pursue this practice which was observed among Chinese elders. Miller and Taylor Piliae (2014), in a review of recent researches on the effects of Tai Chi on cognitive function in community-dwelling older adults, indicated some studies that reported no significant effect of Tai Chi exercise on the performance of the study community. According to them, some of the factors such as the lack of a well designed methodology for performing Tai Chi in the elderly, can cause failure to have appropriate results from this exercise. From the review of results of various studies and their comparison with the results of our current study, it can be said that these contradictory results may be due to the selection of subjects, their gender, physical fitness, history of illness, Tai Chi style, its severity, and duration.

Different forms of Tai Chi exercise which include the style of performance, the duration of exercise and the trainer and his experience are all factors that can influence the outcome of Tai Chi's exercise. Although the duration of intervention and its frequency are mentioned in most studies; however, the time taken by any participant in the study to learn the exercise has not been taken into account, which can be an important factor for the conclusion. In sports like Tai Chi, it's important to use visual senses, imagining, relaxation and remembering the type and sequence of movements which affects the results. Each study has implemented a specific style of Tai Chi exercise; maybe because there is no unique and specific protocol of Tai Chi for the elderlies (Miller \& Taylor Piliae 2014).

Cultural differences also influence the results of intervention by Tai Chi exercise. The familiarity of the elderlies with this sport and its amalgamation with their lives is an important issue (Yeh et al., 2008). More positive results have been reported from the studies conducted in Asian countries, especially China because this exercise is not strange to the elderly with Chinese background and is not in conflict with their lifestyle. While in Iran there is not enough awareness about this sport, which reduces the motivation of the elderly to start and pursue it. This lack of familiarity, made some elderly people think that these movements are useless or do not concentrate enough on the movements of this exercise. Old people living in elderly care centers think that they are worthless, feel themselves closer to death and can lose hope to live. This has led elderly residents of care centers to be considered as the most vulnerable group who experience a lot of stress (Naseh et al. 2014). These factors and continuous stress sometimes make them indifferent, unconcerned or disinterested to engage in activities. According to the study of Kim et al. (2016) on mental-attention effect of Tai Chi on older adults, this exercise should be repeated and practiced in non-familiar cultures, but it was not possible to do it in a privately managed elderly care center. Moreover, the elderly participants in our study did not concentrate enough on the movements of this exercise.

Tai Chi is a complementary therapy, in recent years, non-pharmacological methods have attracted the attention of patients and health care providers (Azimzadeh et al. 2013). In elderly nursing, since a holistic look and attention to all the needs of an elderly person is very much important; therefore, implementation of this exercise can promote the health of the geriatrics and find a clear or effective form for this sport in the elderly population.

One of the limitations of this research was lack of adequate open space for Tai Chi exercise. The research space had no suitable and safe sports hall for the elderly. During the intervention, the elderly who did not have the ability 
to stand up, sat on the chair and made the movements according to their ability and an emphasis on increasing the upper extremity movement. It should be noted that during the exercise of elderly people, there was a chair near each elderly in order to be used in case of tiredness or imbalance. The care givers responsible for each participant in the elderly care centers had to be present near the elderly and help them in the risk of imbalance.

In this study, due to the type of research design (randomized quasi-experimental), generalization of the results of this study to a large extent is not possible; therefore, using the findings of this study should be done with caution.

\section{Acknowledgments}

This paperwas extracted from aMS. thesis approved under clinical trials registration code: IRCT2015072923408N1. The author would like to thank the research branch of the Faculty of Nursing and Midwifery at Iran University of Medical Sciences and the elderly participated in this study for their valuable cooperation. The authors are also grateful to the respected master of Tai Chi, Mrs. Janbaz, who played an important role in acquainting us with the Tai Chi exercise and acquiring the certification.

\section{Conflict of Interest}

The authors declare that they have no conflict of interest.

\section{References}

Askarifard, Gh. R., 2001. [Elderly diseases (Persian)]. Tehran: University of Social Welfare and Rehabilitation Sciences.

Azimzadeh, E., Hosseini, M., \& Nourozi Tabrizi, K., 2013. [Effect of Tai Chi Chuan on quality of life in women with multiple sclerosis (Persian)]. Hayat. 19(2), pp. 1-13

Biglari, N., Arshadi, S., \& Sadeghian, M. R., 2016. [The effect of 8 weeks Taichi Chouan training and walking on cardiovascular disease biomarkers in old women's (Persian)]. Advances in Nursing \& Midwifery, 26(93), pp. 69-76.

Cohen, S., Kamarck, T. \& Mermelstein, R., 1983. A global measure of perceived stress. Journal of Health and Social Behavior, 24(4), pp. 385-96. [DOI:10.2307/2136404]

Hapunda, G. et al., 2015. Living with type 1 diabetes is challenging for Zambian adolescents: Qualitative data on stress, coping with stress and quality of care and life. BMC Endocrine Disorders, 15(1), pp. 2-12. [DOI:10.1186/s12902-015-0013-6]

Harrison, C., et al., 2017. Medical and psychosocial aspects of chronic illness and disability. Burlington: Jones \& Bartlett Learning.
Jacob, L., Breuer, J. \& Kostev, K., 2016. Prevalence of chronic diseases among older patients in German general practices. GMS German Medical Science, 14, pp. 1-7. [DOI:10.3205/000230]

Kim, T. H. M. et al., 2016. The mental-attention Tai Chi effect with older adults. BMC Psychology, 4(1), p. 29. [DOI:10.1186/ s40359-016-0137-0]

Lan, C. et al., 2013. Tai Chi Chuan exercise for patients with cardiovascular disease. Evidence-Based Complementary and Alternative Medicine, 2013, pp. 1-9. [DOI:10.1155/2013/983208]

Lee, Y. M., 2017. The effects of Tai Chi on waist circumference and blood pressure in the elderly. Journal of Physical Therapy Science, 29(1), pp. 172-5. [DOI:10.1589/jpts.29.172]

Lloyd Sherlock, P. et al., 2014. Hypertension among older adults in low- and middle-income countries: prevalence, awareness and control. International Journal of Epidemiology, 43(1), pp. 116-28. [DOI:10.1093/ije/dyt215]

Miller, S. M. \& Taylor Piliae, R. E., 2014. Effects of Tai Chi on cognitive function in community-dwelling older adults: A review. Geriatric Nursing, 35(1), pp. 9-19. [DOI:10.1016/j.gerinurse.2013.10.013]

Naseh, L., Shaikhy, R., \& Rafii, F., 2014. [Quality of life and its related factors among elderlies living in nursing homes (Persian)]. Iran Journal of Nursing, 27(87), pp. 67-78.

Pho, A. T. et al., 2012. Nursing strategies for promoting and maintaining function among community-living older adults: The CAPABLE intervention. Geriatric Nursing, 33(6), pp. 43945. [DOI:10.1016/j.gerinurse.2012.04.002]

Rasel, M., Ardalan, A., 2007. [The future of ageing and its health care costs: A warning for health system (Persian)]. Salmand, 2(2), pp. 300-5.

Sato, S. et al., 2010. Effect of Tai Chi training on baroreflex sensitivity and heart rate variability in patients with coronary heart disease. International Heart Journal, 51(4), pp. 238-41. [DOI:10.1536/ihj.51.238]

Taylor Piliae, R. E., 2014. Tai Ji Quan as an exercise modality to prevent and manage cardiovascular disease: A review. Journal of Sport and Health Science, 3(1), pp. 43-51. [DOI:10.1016/j. jshs.2013.09.002]

Vahedian Azimi, A., et al., 2012. [The relationship between perceived stress and the top five heart disease characteristics in patients with myocardial infarction (Persian)]. Journal of Zanjan University of Medical Sciences, 20(78), pp. 100-12.

Yeh, G.Y. et al., 2008. The effect of Tai Chi exercise on blood pressure: A systematic review. Preventive Cardiology, 11(2), pp. 82-9. [DOI:10.1111/j.1751-7141.2008.07565.x]

Zimmerman, M. E. et al., 2016. Perceived stress is differentially related to hippocampal subfield volumes among older adults. PLOS ONE, 11(5), p.e0154530. [DOI:10.1371/journal. pone.0154530] 